\title{
Crystal structure of (acetonitrile- $N) \operatorname{copper}(\mathrm{I})$ cyanide, $\left[\mathrm{Cu}\left(\mathrm{CH}_{3} \mathrm{CN}\right)\right][\mathrm{CN}]$
}

\author{
H.-L. Keller* and T. Oldag \\ Universität Dortmund, Anorganische Chemie, Otto-Hahn-Str. 6, 44227 Dortmund, Germany
}

Received July 13, 2006, accepted and available on-line September 12, 2006; CCDC no. 1267/1837
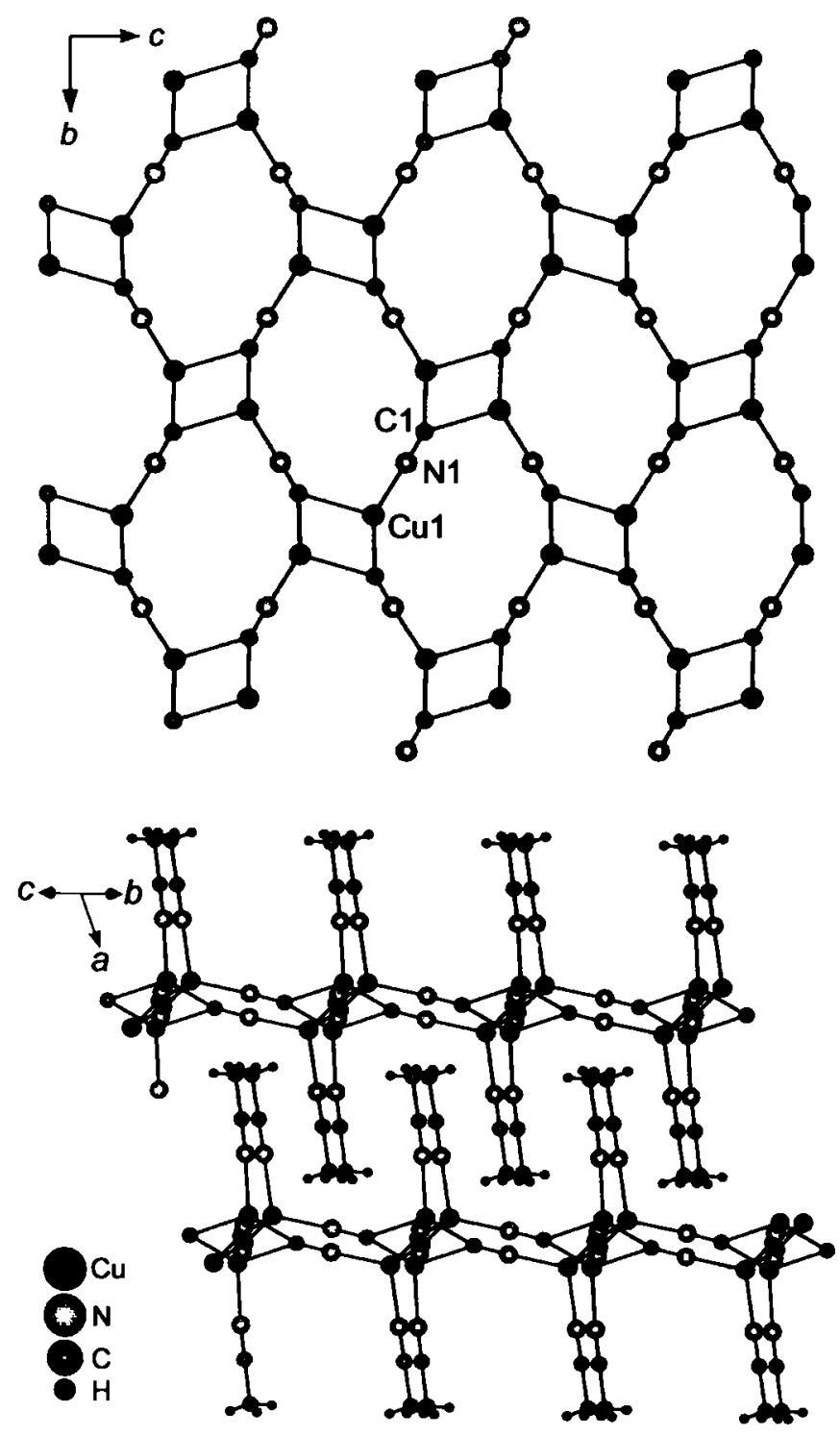

\section{Abstract}

$\mathrm{C}_{3} \mathrm{H}_{3} \mathrm{CuN}_{2}$, monoclinic, $P 12_{1} / c 1$ (no. 14), $a=8.399(2) \AA$, $b=8.144(2) \AA, c=7.836(2) \AA, \beta=116.56(3)^{\circ}, V=479.4 \AA^{3}$, $Z=4, R_{g t}(F)=0.024, w R_{\mathrm{ref}}\left(F^{2}\right)=0.059, T=120 \mathrm{~K}$.

\section{Source of material}

Copper(I) cyanide $(0.2 \mathrm{~g})$ prepared according [1] and acetonitrile (HPLC-grade, $1 \mathrm{~mL}$ ) were mixed together. This mixture was filled in a glass ampule (Duran, interior diameter $10 \mathrm{~mm}$, length $80 \mathrm{~mm}$ ) which was evacuated and sealed. Following annealing

\footnotetext{
*Correspondence author (e-mail: hans-lothar.keller@uni-dortmund.de)
}

was performed in an electric resistance oven at a temperature of $423 \mathrm{~K}$. After three weeks the ampule was taken out, rapidly cooled down to room temperature and the solids were separated from the solvent. By this procedure the title compound can by obtained as single phase.

\section{Discussion}

Hibble et al. have described the rather complex structural chemistry of $\mathrm{CuCN}, \mathrm{AgCN}$ and $\mathrm{AuCN}$ [2]. They were able to refine C and N occupancies of CuCN. The results indicate a 'head-to-tail' disorder of the cyanide groups. We tried to crystallize $\mathrm{CuCN}$ with ordered cyanide groups by solvothermal syntheses with acetonitrile as solvent. Single crystal X-ray structure analysis of the title compound in combination with $\mathrm{X}$-ray powder phase analysis of the bulk material shows that only a solvate of copper(I) cyanide and acetonitrile could be obtained as single phase by the described synthesis. The refinement with the occupancies of $C$ and $\mathbf{N}$ indicates ordered cyanide groups. Two of the three cyanide groups in the coordination sphere of copper are carbon linked to the copper atom, the third one is linked via the nitrogen atom. $\left[\mathrm{Cu}\left(\mathrm{CH}_{3} \mathrm{CN}\right)\right][\mathrm{CN}]$ could be described as a two-dimensional network (Schläfli symbol $8^{2} \cdot 4$; for $\mathrm{Cu}$ and $\mathrm{C}$ ) of condensed $(\mathrm{CuCN})_{4}$ rings perpendicular to [100]. The layers are undulated. Additionally, the copper atom is coordinated by the nitrogen atom of the acetonitrile molecule along [100]. A part of a single layer of the two-dimensional network is shown in the top of the figure, the interlocking of this layers by the acetonitrile molecules at the bottom. Plane $[3,4]$ and undulated layers $[5,6]$ of $(\mathrm{CuCN})_{6}$ rings or $(\mathrm{CuCN})_{8}$ rings [7] are already known. Layers built of $(\mathrm{CuCN})_{4}$ rings are less common [8].

Table 1. Data collection and handling.
Crystal:

Wavelength:

$\mu:$

Diffractometer, scan mode:

$2 \theta_{\text {max: }}$

$N(h k l)_{\text {measured, }} N(h k l)_{\text {unique: }}$

Criterion for $I_{\mathrm{obs}}, N(h k l)_{\mathrm{gt}}$ :

$N($ param) refined:

Programs: colorless thick plate,

size $0.10 \times 0.17 \times 0.25 \mathrm{~mm}$

Mo $K_{\alpha}$ radiation $(0.71073 \AA)$

$43.85 \mathrm{~cm}^{-1}$

Nonius KappaCCD, $\omega$

$60.22^{\circ}$

10254,1402

$I_{\text {obs }}>2 \sigma\left(I_{\text {obs }}\right), 1216$

67

SHELXTL-Plus [9], DIAMOND [10]
Table 2. Atomic coordinates and displacement parameters (in $\AA^{2}$ ).

\begin{tabular}{llllll}
\hline Atom & Site & $x$ & $y$ & $z$ & $U_{\text {iso }}$ \\
\hline $\mathbf{H}(1)$ & $4 e$ & $0.208(5)$ & $0.943(4)$ & $0.218(5)$ & $0.046(9)$ \\
$\mathrm{H}(2)$ & $4 e$ & $0.238(6)$ & $0.874(6)$ & $0.386(6)$ & $0.07(1)$ \\
$\mathrm{H}(3)$ & $4 e$ & $0.235(6)$ & $1.047(5)$ & $0.366(6)$ & $0.07(1)$ \\
\hline
\end{tabular}


Table 3. Atomic coordinates and displacement parameters (in $\AA^{2}$ ).

\begin{tabular}{|c|c|c|c|c|c|c|c|c|c|c|}
\hline Atom & Site & $x$ & $y$ & $z$ & $U_{11}$ & $U_{22}$ & $U_{33}$ & $U_{12}$ & $U_{13}$ & $U_{23}$ \\
\hline $\mathrm{Cu}(1)$ & $4 e$ & $0.60219(3)$ & $0.93220(3)$ & $0.14604(4)$ & $0.0194(1)$ & $0.0144(1)$ & $0.0196(1)$ & $0.0014(1)$ & $0.0106(1)$ & $0.0024(1)$ \\
\hline $\mathbf{N}(1)$ & $4 e$ & $0.5506(2)$ & $0.7505(2)$ & $0.2762(3)$ & $0.0235(9)$ & $0.0184(8)$ & $0.0195(8)$ & $0.0014(7)$ & $0.0108(7)$ & $0.0009(7)$ \\
\hline $\mathbf{N}(2)$ & $4 e$ & $0.8659(3)$ & $0.9475(2)$ & $0.2340(3)$ & $0.0213(9)$ & $0.030(1)$ & $0.026(1)$ & $-0.0010(8)$ & $0.0102(8)$ & $0.0006(8)$ \\
\hline$C(1)$ & $4 e$ & $0.5142(3)$ & $0.6437(3)$ & $0.3479(3)$ & $0.023(1)$ & $0.016(1)$ & $0.024(1)$ & $0.0031(8)$ & $0.0137(9)$ & $0.0009(8)$ \\
\hline$C(2)$ & $4 e$ & $1.0137(3)$ & $0.9510(3)$ & $0.2767(3)$ & $0.023(1)$ & $0.028(1)$ & $0.022(1)$ & $-0.0016(9)$ & $0.0103(9)$ & $-0.0001(9)$ \\
\hline$C(3)$ & $4 e$ & $0.2033(4)$ & $0.9537(5)$ & $0.3302(5)$ & $0.018(1)$ & $0.049(2)$ & $0.042(2)$ & $-0.002(1)$ & $0.013(1)$ & $-0.001(1)$ \\
\hline
\end{tabular}

\section{References}

1. Barber, H. J.: Cuperus Cyanide: A Note on its Preparation and Use. J. Chem. Soc. 26 (1943) 79-80.

2. Hibble, S. J.; Eversfield, S. G.; Cowley, A. R.; Chippindale, A. M.: Copper(I) Cyanide: A Simple Compound with a Complicated Structure and Surprising Room-Temperature Reactivity. Angew. Chem. 116 (2004) 638-640.

3. Colacio, E.; Kievekas, R.; Loret, F.; Sunberg, M.; Suarez-Varela, J.; Bardaij, M.; Laguna A.: Architecture Dependence on the Steric Constrains of the Ligand in Cyano-Bridged Copper(I) and Copper(II)-Copper(I) Mixed-Valence Polymer Compounds Containing Diamines: Crystal Structures and Spectroscopic and Magnetic Properties. Inorg. Chem. 41 (2002) 5141-5149.

4. Thao, Y.; Hong, M.; Su, W.; Cao, R.; Zhou, Z.; Cahn, A. S. C.: Synthesis and characterization of two copper cyanide complexes with hexagonal $\mathrm{Cu}_{6}$ units. J. Chem. Soc., Dalton Trans. (2000) 1685-1686.
5. Cromer, D. T.; Larson, A. C.: The crystal structure of $\mathrm{KCu}_{2}(\mathrm{CN})_{3} \cdot \mathrm{H}_{2} \mathrm{O}$. Acta Crystallogr. 15 (1962) 397-403.

6. Chesnut, D. J.; Kusnetzow, A.; Birge, R.; Zubieta, J.: Ligand Influences on Copper Cyanide Solid-State Architecture: Flattened and Fused "Slinky", Corrugated Sheet, and Ribbon Motifs in the Copper-CyanideTriazolate-Organoamine Family. Inorg. Chem. 38 (1999) 5484-5494.

7. Bowmaker, G.A.; Harti, H.; Urban, V.: Orystal Structures and Vibrational Spectroscopy of $[\mathrm{NBu}][\mathrm{Cu}(\mathrm{CN}) X], \boldsymbol{X}=\mathrm{B}, \mathrm{I})$ and $\left[\mathrm{NBu}_{4}\right]\left[\mathrm{Cu}_{3}(\mathrm{CN})_{4}\right] \cdot \mathrm{CH}_{3} \mathrm{CN}$. Inorg. Chem. 39 (2000) 4548-4554.

8. Pretsch, T.; Brüdgam, L.; Hartl, H.: Conformation and cross linking of $(\mathrm{CuCN})_{6}$-rings in polymeric cyanocuprates $(\mathrm{I}) n\left[\mathrm{Cu}_{2}(\mathrm{CN})_{3}\right](n=2,3) . \mathrm{Z}$. Anorg. Allg. Chem. 630 (2004) 353-360.

9. Sheldrick, G. M.: SHELXTL-Plus. Structure Determination Software Suite. Bruker AXS, Madison, Wisconsin, USA 1998.

10. Brandenburg, K: DIAMOND. Visual Crystal Structure Information System. Version 2.1e. Crystal Impact, Bonn, Germany 2005. 
(C) by Oldenbourg Wissenschaftsverlag, München

\title{
Crystal structure of dicyano( $N$-(1-iminoethyl)acetamidine)palladium(II), $\operatorname{Pd}(\mathrm{CN})_{2}\left(\mathrm{C}_{4} \mathrm{H}_{9} \mathbf{N}_{3}\right)$
}

\author{
H.-L. Keller* and T. Oldag
}

Universität Dortmund, Anorganische Chemie, Otto-Hahn-Str. 6, 44227 Dortmund, Germany

Received July 13, 2006, accepted and available on-line September 12, 2006; CCDC no. 1267/1838
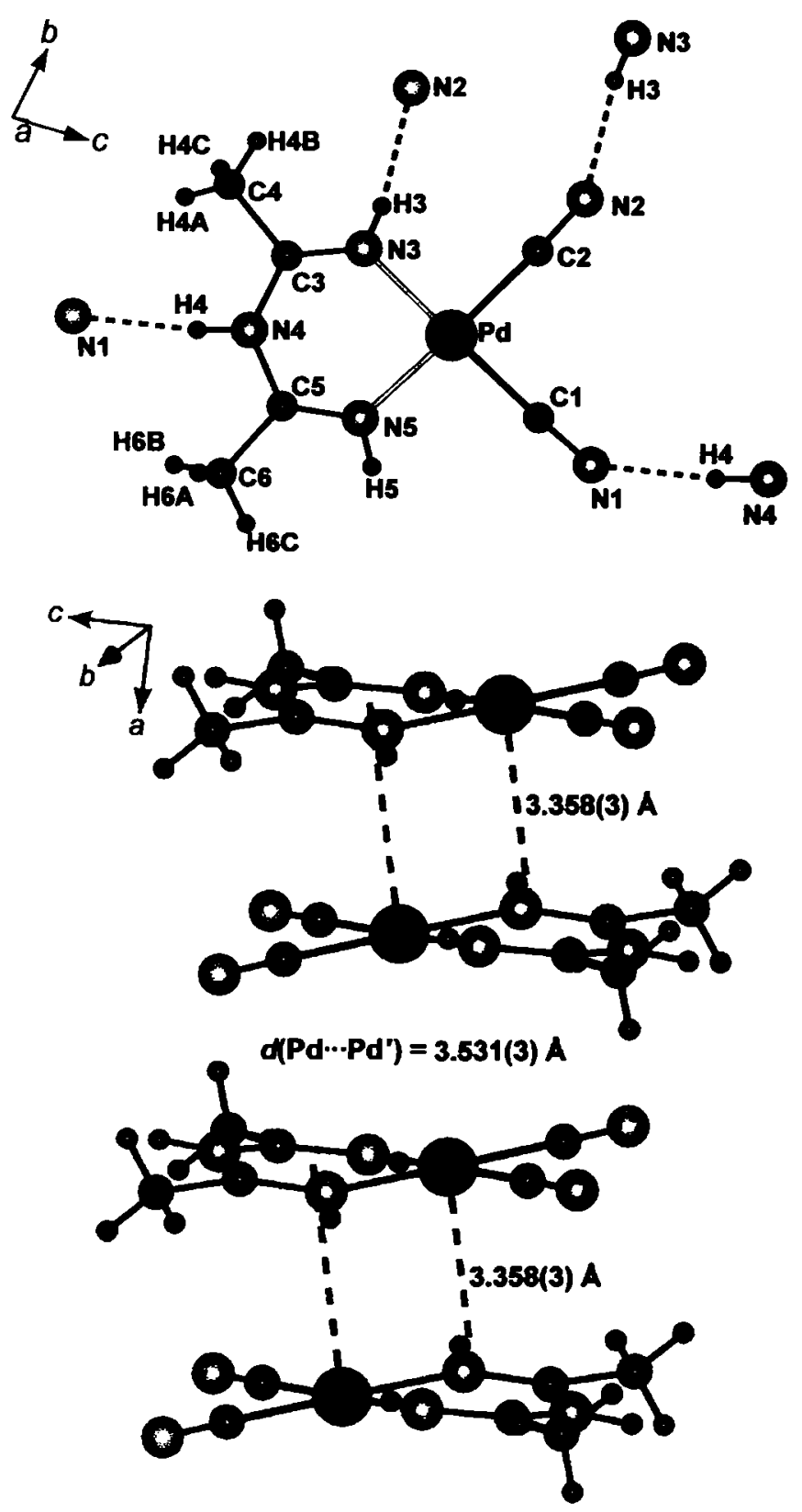

Abstract

$\mathrm{C}_{6} \mathrm{H}_{9} \mathrm{~N}_{5} \mathrm{Pd}$, triclinic, $P \overline{1}$ (no. 2$), a=6.906(1) \AA$,

$b=7.371(2) \AA, c=8.784(2) \AA, \alpha=80.67(3)^{\circ}, \beta=82.27(3)^{\circ}$,

$\gamma=73.30(3)^{\circ}, V=420.7 \AA^{3}, Z=2, R_{g t}(F)=0.057$, $w R_{\text {ref }}\left(F^{2}\right)=0.078, T=150 \mathrm{~K}$.

*Correspondence author (e-mail: hans-lothar.keller@uni-dortmund.de)

\section{Source of material}

Palladium(II) cyanide ( 0.2 g) prepared according [1] and acetonitrile (HPLC-grade, $1 \mathrm{~mL}$ ) were mixed together. The mixture was filled in a glass ampule (Duran, interior diameter $10 \mathrm{~mm}$, length $80 \mathrm{~mm}$ ) which was evacuated and sealed. Subsequent annealing was performed in an electric resistance oven at a temperature of $393 \mathrm{~K}$. After 18 months the ampule was taken out, rapidly cooled down to room temperature and the precipitate was separated from the solvent. It contained several colorless crystals of the title compound and micro crystalline $\operatorname{Pd}(C N)_{2}$.

\section{Discussion}

The main idea of the reported syntheses was the recrystallization of amorphous $\mathrm{Pd}(\mathrm{CN})_{2}$ using acetonitrile as solvent under solvothermal conditions. The structure analysis of the title compound shows that the trimerization of acetonitrile leads to $N$-(1-iminoethyl)acetamidine that is linked to palladium. Trimerizations of nitriles catalyzed by metals of the third main group (Al, $\mathrm{Ga}, \mathrm{In}$ ) are already known. In contradiction to these trimerization reactions described by Kukushkin \& Pombeiro [2] the organic part of the title compound shows no CN group at position 3 .

The interatomic distances of the triazapentadiene $(d(\mathrm{~N} 5-\mathrm{C5})=$ $1.294(1) \AA, d(\mathrm{C} 5-\mathrm{N} 4)=1.363(1) \AA, d(\mathrm{~N} 4-\mathrm{C} 3)=1.379(1) \AA$, $d(\mathrm{C} 3-\mathrm{N} 3)=1.274(1) \AA)$ are shorter than typical $\mathrm{C}-\mathrm{N}$ single bonds but longer than typical double bonds. As a conclusion of this distance observations the six-membered ring (Pd, C3, N3, N4, $\mathrm{N} 5, \mathrm{C5}$ ) seems to be a quasi-aromatic system (figure, top). The unit cell contains two planar ( $N$-(1-iminoethyl)acetamidine)palladium(II) cyanide groups in between these molecules a symmetry center $(1 / 2,1 / 2,1 / 2)$ is located. We guess that an interaction of the $d_{2}{ }^{2}$ orbital of the palladium atom with the quasi-aromatic triazapentadiene-palladium ring stabilizes the crystal packing along the $[100]$ direction $(d(P d \cdots$ - ring $)=3.358(3) \AA$ and 3.777(3) $\AA$; figure, bottom). Additionally, the ( $N$-(1-iminoethyl)acetamidine)palladium(II) cyanide units are interlinked through hydrogen bonds along [010] and [001] building a double chain as shown in the figure, top, $(d(\mathrm{H} 3 \cdots \mathrm{N} 2)=2.30(6) \AA, d(\mathrm{H} 4 \cdots \mathrm{N} 1)=2.04(6) \AA)$.

Table 1. Data collection and handling.

Crystal:

Wavelength:

$\mu$ :

Diffractometer, scan mode:

$2 \theta_{\max }$ :

$N(h k l)_{\text {measured }} N(h k l)_{\text {unique: }}$

Criterion for $I_{\mathrm{obs}}, N(\mathrm{hkl})_{\mathrm{gt}}$ :

$N($ param) refined:

Programs: colorless fragment, size $0.1 \times 0.3 \times 0.5 \mathrm{~mm}$ Mo $K_{a}$ radiation ( $0.71073 \AA$ )

$21.55 \mathrm{~cm}^{-1}$

Nonius KappaCCD, $\omega$

$59.98^{\circ}$

6849,2315

$I_{\mathrm{obs}}>2 \sigma\left(I_{\mathrm{obs}}\right), 1593$

136

SHELXTL-PIus [3], DIAMOND [4] 
Table 2. Atomic coordinates and displacement parameters (in $\AA^{2}$ ).

\begin{tabular}{llllll}
\hline Atom & Site & $x$ & $y$ & $z$ & $U_{\text {iso }}$ \\
\hline H(3) & $2 i$ & $0.597(7)$ & $0.783(7)$ & $0.344(6)$ & 0.020 \\
H(4) & $2 i$ & $0.813(7)$ & $0.383(7)$ & $0.107(6)$ & 0.022 \\
H(5) & $2 i$ & $0.795(7)$ & $0.189(7)$ & $0.521(6)$ & 0.022 \\
H(4A) & $2 i$ & $0.588(7)$ & $0.677(7)$ & $0.006(6)$ & 0.030 \\
H(4B) & $2 i$ & $0.579(7)$ & $0.849(8)$ & $0.080(6)$ & 0.030 \\
H(4C) & $2 i$ & $0.761(8)$ & $0.760(7)$ & $0.015(6)$ & 0.030 \\
H(6A) & $2 i$ & $0.784(7)$ & $0.066(7)$ & $0.188(6)$ & 0.026 \\
H(6B) & $2 i$ & $0.989(8)$ & $0.060(7)$ & $0.206(6)$ & 0.026 \\
H(6C) & $2 i$ & $0.865(7)$ & $-0.021(7)$ & $0.348(6)$ & 0.026 \\
\hline
\end{tabular}

Table 3. Atomic coordinates and displacement parameters (in $\AA^{2}$ ).

\begin{tabular}{|c|c|c|c|c|c|c|c|c|c|c|}
\hline Atom & Site & $x$ & $y$ & $z$ & $U_{11}$ & $U_{22}$ & $U_{33}$ & $U_{12}$ & $U_{13}$ & $U_{23}$ \\
\hline $\operatorname{Pd}(1)$ & $2 i$ & $0.73677(6)$ & $0.53636(6)$ & $0.55088(5)$ & $0.0201(2)$ & $0.0135(2)$ & $0.0116(2)$ & $-0.0024(2)$ & $-0.0014(1)$ & $-0.0035(1)$ \\
\hline $\mathbf{N}(1)$ & $2 i$ & $0.8318(6)$ & $0.3333(6)$ & $0.8826(5)$ & $0.032(3)$ & $0.025(3)$ & $0.019(3)$ & $-0.004(2)$ & $-0.007(2)$ & $-0.005(2)$ \\
\hline$N(2)$ & $2 i$ & $0.6461(7)$ & $0.9287(6)$ & $0.6715(5)$ & $0.036(3)$ & $0.020(3)$ & $0.020(3)$ & $0.000(2)$ & $-0.007(2)$ & $-0.004(2)$ \\
\hline$N(3)$ & $2 i$ & $0.6666(6)$ & $0.6754(6)$ & $0.3395(5)$ & $0.017(2)$ & $0.014(2)$ & $0.015(2)$ & $0.003(2)$ & $0.001(2)$ & $-0.004(2)$ \\
\hline $\mathbf{N}(4)$ & $2 i$ & $0.7832(6)$ & $0.4170(6)$ & $0.1996(5)$ & $0.028(2)$ & $0.014(2)$ & $0.013(2)$ & $-0.004(2)$ & $0.000(2)$ & $-0.005(2)$ \\
\hline$N(5)$ & $2 i$ & $0.7928(6)$ & $0.2904(6)$ & $0.4598(5)$ & $0.026(3)$ & $0.011(2)$ & $0.016(3)$ & $-0.005(2)$ & $-0.002(2)$ & $0.000(2)$ \\
\hline C(1) & $2 \boldsymbol{i}$ & $0.7991(7)$ & $0.4057(7)$ & $0.7586(6)$ & $0.017(3)$ & $0.018(3)$ & $0.025(3)$ & $-0.003(2)$ & $-0.002(2)$ & $-0.012(3)$ \\
\hline$C(2)$ & $2 i$ & $0.6810(7)$ & $0.7810(8)$ & $0.6321(6)$ & $0.018(3)$ & $0.025(3)$ & $0.014(3)$ & $-0.004(3)$ & $-0.003(2)$ & $0.000(3)$ \\
\hline$c(3)$ & $2 i$ & $0.7009(7)$ & $0.6090(7)$ & $0.2104(6)$ & $0.018(3)$ & $0.015(3)$ & $0.022(3)$ & $-0.004(2)$ & $-0.003(2)$ & $-0.002(2)$ \\
\hline$C(4)$ & $2 i$ & $0.6543(9)$ & $0.7324(8)$ & $0.0603(7)$ & $0.032(3)$ & $0.019(3)$ & $0.022(3)$ & $-0.003(3)$ & $-0.005(3)$ & $-0.005(3)$ \\
\hline$C(5)$ & $2 i$ & $0.8128(7)$ & $0.2668(7)$ & $0.3151(6)$ & $0.014(3)$ & $0.017(3)$ & $0.021(3)$ & $-0.004(2)$ & $-0.002(2)$ & $-0.005(2)$ \\
\hline$C(6)$ & $2 i$ & $0.8726(9)$ & $0.0756(8)$ & $0.2614(7)$ & $0.028(3)$ & $0.018(3)$ & $0.020(3)$ & $-0.007(3)$ & $0.000(3)$ & $-0.003(2)$ \\
\hline
\end{tabular}

\section{References}

1. Bigelow, J. H.: Potassium Tetracyanopalladate(II). Inorg. Syn. II (1946) 245-250.

2. Kukushkin, V. Y.; Pombeiro, A. J. L.: Additions to Metal-Activated Organonitriles. Chem. Rev. 102 (2002) 1771-1802.
3. Sheldrick, G. M.: SHELXTL-Plus. Structure Determination Software Suite. Bruker AXS, Madison, Wisconsin, USA 1998.

4. Brandenburg, K: DIAMOND. Visual Crystal Structure Information System. Version 2.1e. Crystal Impact, Bonn, Germany 2005. 